\title{
Orientation-based Continuum Damage Models for Rocks
}

\author{
Xiaoyu Liu, ${ }^{1}$ Xiangchu Yin, $^{2,3}$ and Naigang Liang ${ }^{2}$
}

\begin{abstract}
A general formulation of the Helmholtz free energy used in thermodynamics of damage process of rocks is derived within a multi-scale framework. Such a physically-based thermodynamic state potential has a hybrid, discrete/continuum, nature in the sense that it adopts a continuum description but subsumes the statistical ensemble average of the action of the entirety of microscopic degrees of freedom. The choice of the relevant damage variables results therefore directly from the breaking of contact cohesive bonds, and it naturally obeys the Clausius-Duhem inequality. Furthermore, motivated by the fact that the free energy is formulated by the integral of potentials independently defined on different orientations over the upper hemisphere, the damage evolution equation is formulated on a generic orientation. Consequently, the mechanical behavior of a rock material generally becomes anisotropic characteristics in the inelastic regime even if the material is initially isotropic, thus introducing dissipation-induced anisotropy in a very natural and simple way. Finally, the development of the lattice solid model can be cast into the framework of the orientation based continuum constitutive model.
\end{abstract}

Key words: Multi-scale modeling, thermodynamics, constitutive relation, damage, lattice solid model.

\section{Introduction}

As a naturally discontinuous, anisotropic and inhomogeneous geological material, the rocks are assemblages of blocks formed by connected fractures, and the interactions between the rock blocks and fractures (interfaces between blocks) are the main factors affecting the mechanical behavior of the fractured rock masses. Therefore the discrete approach, represented mostly at present by the distinct element method (DEM) and discontinuous deformation analysis (DDA) (JING, 1998), has become prominent as a tool for providing more realistic representation of problem geometry of fractured rocks since fractures are explicitly represented, both geometrically and mechanically. Nevertheless, this advantage entails sacrificing the problem size since representations of individual blocks and fractures put large demands on computer memory and speed. Therefore, despite the popularity of the

\footnotetext{
${ }^{1}$ Division of Engineering Sciences, Institute of Mechanics, Chinese Academy Sciences, Beijing 100080, China. E-mail: liuxy@imech.ac.cn

${ }^{2}$ LNM, Institute of Mechanics, Chinese Academy Sciences, Beijing 100080, China.

${ }^{3}$ Center of Analysis and Prediction, China Earthquake Administration, Beijing 100036, China.
} 
'discrete approach', its applicability to solve practical problems remains a challenging task.

Alternatively, the ultimate in reduction of fractured rock masses is represented by continuum field theory where all discrete degrees of freedom are replaced by continuous field variables obeying macroscopic differential equations. Because details of blocks and fracture systems are coarse grained out of the formulation, continuum models are not subject to length and time scale limitations, and the material parameters entering the models such as stresses and displacements are suited directly to measurements and senses. The constitutive relations, however, are often empirically determined, making their reliability over a wide range of deformations dubious.

The purpose of the current paper is, on one hand, to focus on multi-scale constitutive modeling for rocks. In this context, a multi-scale modeling methodology is presented naturally to combine the interactions of discrete micro-structures of a rock material on the small scale with the usual framework of continuum mechanics on the very large scale. By using such micromechanics theory, we develop an orientation-based continuum damage model having the following virtues: (1) the Helmholtz free energy is calculated exclusively from the microscopic contact constitutive relationship, the discrete micro-structures and the macroscopic kinematical variables without empirical phenomenological assumption; (2) the microscopic formation of damage is directly the result of the breaking of cohesive bonds, and it naturally obeys the laws of thermodynamics; (3) damage-induced anisotropy is incorporated into the constitutive model in a natural and simple way.

On the other hand, based on the fact that the free energy is expressed as the integral of potentials defined on different orientation over the upper hemisphere, and with the help of the damage evolution equations formulated on a generic orientation, the lattice solid model (NAPIER and Dede, 1997; Mora and Place, 1999; Li et al., 2000), which has been used to simulate fracture initiation and propagation in rocks as an attractive alternative to finite-element analysis, can be derived from the directional discretization of the continuum constitutive model. Consequently, the orientation-based constitutive model not only can be numerically implemented through the lattice solid model, but also offers a unified framework for the development of the lattice solid model.

Symbolic and index notations are used in the present paper; for instance, the strain tensor is denoted by either $\boldsymbol{E}$ or $E_{i j}$. In the symbolic notation, '.' and ' $:$ ' stand for the first- and second-order contractions, ' $\otimes$ ' denotes the tensor product.

\section{Multi-scale Modeling of Rocks}

Consider a rock mass consisting of abstract material points on the macroscopic scale indicated in Figure 1(a). On the microscopic scale, an infinitesimal neighbor- 


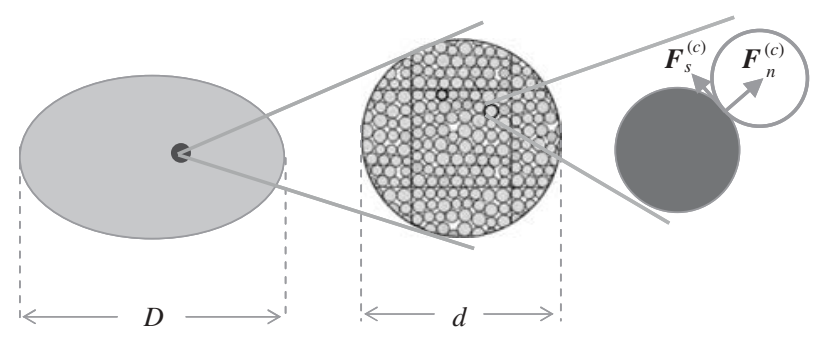

(a)

(b)

(c)

Figure 1

A rock body consisting of discrete block ensembles (RVEs): (a) Macrostructure, (b) microstructure and (c) contact blocks.

hood of a material point, namely the so-called representative volume element, is associated with an ensemble of discrete blocks formed by connected fractures, cf. Figure 1(b). Those blocks with sufficient large numbers are idealized as rigid elements in this paper. Denote the material configuration of the solid by $A$ and the configuration at the present time $\tau$ by $B$. The distance vectors between a pair of adjacent elements $a$ and $b$ in configuration $A$ and configuration $B, X^{(a, b)}$ and $\boldsymbol{x}^{(a, b)}$, are written as

$$
\boldsymbol{X}^{(a, b)}=\boldsymbol{X}^{(a)}-\boldsymbol{X}^{(b)}, \quad \boldsymbol{x}^{(a, b)}=\boldsymbol{x}^{(a)}-\boldsymbol{x}^{(b)}, \quad \text { with } \quad \boldsymbol{X}^{(a)}-\boldsymbol{X}^{(b)}=R^{(a, b)} \boldsymbol{N}^{(a, b)},
$$

where $R^{(a, b)}$ and $\boldsymbol{N}^{(a, b)}$ are the length and unit vector of $\boldsymbol{X}^{(a, b)}$, respectively.

As shown in Figure 1(c), the contact actions among adjacent elements are assumed to be concentrated at the contact points. The local stress in the contact region between elements $a$ and $b$ thus is replaced by concentrated force at the contact point $c$

$$
\boldsymbol{F}^{(c)}=\boldsymbol{F}_{n}^{(c)}+\boldsymbol{F}_{s}^{(c)}
$$

where $\boldsymbol{F}_{n}^{(c)}$ is the normal force, and $\boldsymbol{F}_{s}^{(c)}$ is the shear force, while the local strain is replaced by relative displacement

$$
\boldsymbol{u}^{(c)}=\boldsymbol{u}^{(a)}-\boldsymbol{u}^{(b)}=\boldsymbol{u}_{n}^{(c)}+\boldsymbol{u}_{s}^{(c)},
$$

where the normal relative displacement, $\boldsymbol{u}_{n}^{(c)}$, and the shear relative displacement, $\boldsymbol{u}_{s}^{(c)}$, are given by, respectively,

$$
\begin{aligned}
& \boldsymbol{u}_{n}^{(c)}=\left(\boldsymbol{u}^{(a)}-\boldsymbol{u}^{(b)}\right) \cdot\left(\boldsymbol{N}^{(a, b)} \otimes \boldsymbol{N}^{(a, b)}\right), \\
& \boldsymbol{u}_{s}^{(c)}=\left(\boldsymbol{u}^{(a)}-\boldsymbol{u}^{(b)}\right) \cdot\left(\boldsymbol{I}-\boldsymbol{N}^{(a, b)} \otimes \boldsymbol{N}^{(a, b)}\right) .
\end{aligned}
$$

As response functions for the contact actions, the following two types of linear elastic contact constitutive relations 


$$
\boldsymbol{F}_{n}^{(c)}=K^{(n)} \boldsymbol{u}_{n}^{(c)}, \quad \boldsymbol{F}_{s}^{(c)}=K^{(s)} \boldsymbol{u}_{s}^{(c)}
$$

are considered, in which $K^{(n)}, K^{(s)}$ are the respective elastic constants in directions normal and tangential to the contact. Correspondingly, the mean energy of the contact actions within the RVE in configuration $B$ takes the form

$$
\begin{aligned}
e & =\frac{1}{V} \sum_{<a, b>} W\left(\boldsymbol{u}^{(a)}-\boldsymbol{u}^{(b)}\right) \\
& =\frac{1}{2 V} \sum_{<a, b>}\left[\left(K^{(n)}-K^{(s)}\right)\left(\left(\boldsymbol{u}^{(a)}-\boldsymbol{u}^{(b)}\right) \cdot \boldsymbol{N}^{(a, b)}\right)^{2}+K^{(s)}\left(\boldsymbol{u}^{(a)}-\boldsymbol{u}^{(b)}\right) \cdot\left(\boldsymbol{u}^{(a)}-\boldsymbol{u}^{(b)}\right)\right] .
\end{aligned}
$$

Here $V$ is the volume of the RVE, $\langle a, b\rangle$ runs over all the contacts within the RVE.

In order to construct a continuum model on the macroscopic scale based on the discrete model on the microscopic scale, the crucial step lies in establishing a correspondence between the discrete and the continuum kinematical descriptors. For that, the deformation of element $a$ within the RVE is split into two parts. In the first part the centroid $\boldsymbol{X}$ of the RVE moves to a spatial position $\boldsymbol{x}$ in a vector of macrodisplacements $\boldsymbol{u}$, while in the second, element $a$ having a relative position $\Xi^{(a)}$ with respect to the centroid moves to a new relative spatial position $\xi^{(a)}$ in a vector of micro-displacements $v^{(a)}$, cf. Figure 2. Following the assumption that the size of the RVE is assumed to be conceptually infinitesimal on the macroscopic scale, but is much larger than the size of discrete blocks on the microscopic scale, the relative position $\Xi^{(a)}$ of the element $a$ to the centroid of the "RVE", which is not necessarily small on the microscopic scale, must be small from the macroscopic view of point. As a result, the displacement of element $a$ can be approximated by a polynomial expansion, i.e.,

$$
\begin{aligned}
& \boldsymbol{u}^{(a)}\left(\boldsymbol{x}^{(a)}\right)=\boldsymbol{u}\left(\boldsymbol{x}+\boldsymbol{\Xi}^{(a)}\right) \\
& \quad=\boldsymbol{u}(\boldsymbol{x})+\left(\boldsymbol{u}(\boldsymbol{x}) \otimes \nabla_{X}\right) \cdot \boldsymbol{\Xi}^{(a)}+\frac{1}{2}\left(\boldsymbol{u}(\boldsymbol{x}) \otimes \nabla_{X} \otimes \nabla_{X}\right):\left(\boldsymbol{\Xi}^{(a)} \otimes \boldsymbol{\Xi}^{(a)}\right)+\cdots,
\end{aligned}
$$

where $\nabla_{X}$ denotes the gradient operator with respect to $X$. When constructing a continuum model accounting for short-range interactions, it suffices to consider homogeneous deformations, which implies the second and higher order derivatives of the displacement field can be neglected. In this case, the above equation becomes

$$
\begin{aligned}
& \boldsymbol{u}^{(a)}\left(\boldsymbol{x}^{(a)}\right) \approx \boldsymbol{u}(\boldsymbol{x})+\left(\boldsymbol{u}(\boldsymbol{x}) \otimes \nabla_{\boldsymbol{X}}\right) \cdot \Xi^{(a)} \\
& =\boldsymbol{u}(\boldsymbol{x})+\left[\frac{1}{2}\left(\boldsymbol{u}(\boldsymbol{x}) \otimes \nabla_{\boldsymbol{X}}+\nabla_{\boldsymbol{X}} \otimes \boldsymbol{u}(\boldsymbol{x})\right)+\frac{1}{2}\left(\boldsymbol{u}(\boldsymbol{x}) \otimes \nabla_{X}-\nabla_{\boldsymbol{X}} \otimes \boldsymbol{u}(\boldsymbol{x})\right)\right] \cdot \Xi^{(a)},
\end{aligned}
$$

where the displacement gradient $u_{i j}$ is decomposed into a symmetric part and an antisymmetric part. In many homogenization studies on granular assemblies the 


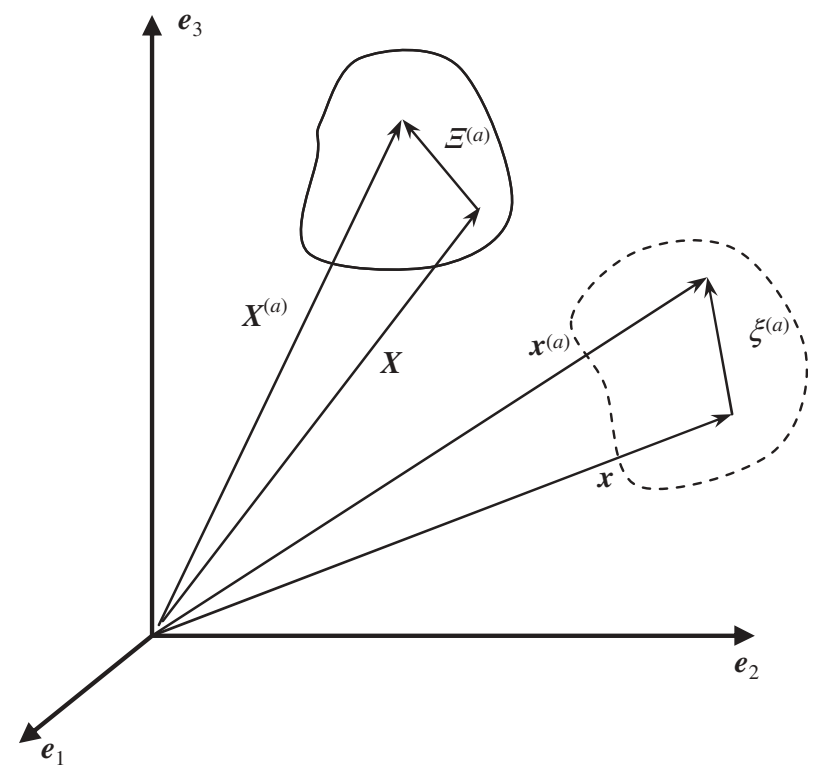

Figure 2

Schematic of the deformation of element $a$ within the RVE.

contribution of the antisymmetric part is neglected (CHRISTOFFERSON et al., 1981; Walton, 1987; BAthurst and Rothenburg, 1988). Accordingly, the relative displacement vector between elements $a$ and $b$ is rewritten as

$$
\boldsymbol{u}^{(a)}-\boldsymbol{u}^{(b)} \approx E(\boldsymbol{x}) \cdot\left(\boldsymbol{X}^{(a)}-\boldsymbol{X}^{(b)}\right), \quad \boldsymbol{E}(\boldsymbol{x})=\frac{1}{2}\left(\boldsymbol{u}(\boldsymbol{x}) \otimes \nabla_{\boldsymbol{X}}+\nabla_{\boldsymbol{X}} \otimes \boldsymbol{u}(\boldsymbol{x})\right),
$$

where $\boldsymbol{E}$ is the Lagrangean strain tensor. By substituting Eq. (9) into Eq. (6) and making use of Eq. (1), the energy density of the discrete system, expressed in index notation, is as follows

$$
\begin{aligned}
e= & \frac{1}{V} \sum_{<a, b>} W\left(R^{(a, b)} \boldsymbol{E} \cdot \boldsymbol{N}^{(a, b)}\right) \\
= & \frac{\left(K^{(n)}-K^{(s)}\right) E_{i j} E_{s t}}{2 V} \sum_{<a, b>}\left(R^{(a, b)}\right)^{2} N_{i}^{(a, b)} N_{j}^{(a, b)} N_{s}^{(a, b)} N_{t}^{(a, b)} \\
& +\frac{K^{(s)} E_{i j} E_{s t}}{8 V} \sum_{<a, b>}\left(R^{(a, b)}\right)^{2}\left(\delta_{i s} N_{j}^{(a, b)} N_{t}^{(a, b)}+\delta_{i t} N_{j}^{(a, b)} N_{s}^{(a, b)}\right. \\
& \left.+\delta_{j s} N_{i}^{(a, b)} N_{t}^{(a, b)}+\delta_{j t} N_{i}^{(a, b)} N_{s}^{(a, b)}\right) .
\end{aligned}
$$

Furthermore, we require that the power stored within the RVE is equal to the total energy spent over the material neighborhood occupying the same region. As 
consequences of the energy equivalence between micro and macro scale, the Helmholtz free energy without energy dissipation is calculated exclusively from the microscopic contact constitutive relationship, the discrete micro-structures and the macroscopic kinematical variables, and does not involve an empirical phenomenological assumption.

\section{Orientation-based Continuum Constitutive Model}

\subsection{Elastic Behavior}

Before moving on to the more complex irreversible nonlinear behavior of rocks, it is worth describing the material response without energy dissipation. In terms of Fung's (FunG, 1969) classification of the different forms of elastic model, all nondissipative materials are required to be hyperelastic since this is the only means by which the material is guaranteed to obey the First Law of Thermodynamics. Because of the fact that the hyperelastic behavior is entirely defined by a Helmholtz free energy function, a physical and general constitutive law can be obtained by virtue of Eq. (10). The stresses are given by

$$
\sigma_{i j}=\frac{\partial e}{\partial E_{i j}}
$$

and the tangent stiffness tensor obtained by further differentiation

$$
C_{i j s t}=\frac{\partial^{2} e}{\partial E_{i j} \partial E_{s t}} .
$$

Since the energy density in Eq. (10) is formulated by a summation form, the calculation of the constitutive laws thus need to extend over all the relative distance vectors within the RVE. For a material with randomly distributed geometries of discrete microstructures, the calculation is often unfeasible and unnecessary. It becomes necessary to replace the summation setting in Eq. (10) by its continuum surrogates. The latter removes the discrete degrees of freedom but subsumes the statistical ensemble average of the action of the entirety of microscopic degrees of freedom. For that, select a spherical coordinate system $(R, \theta, \phi)$ such that a distance vector is characterized by a corresponding position in the upper hemispherical coordinate system. ${ }^{1}$ As a result, the discrete summation expression of Eq. (10) can be translated into an integral form by

\footnotetext{
${ }^{1}$ Because the potential energy density is independent of the direction of the distance vector, we herein require the distance vector to be oriented in the upper half spherical coordinate system.
} 


$$
\begin{aligned}
e= & \int_{0}^{2 \pi} \int_{0}^{\pi / 2} \int_{R_{1}}^{R_{2}} D(R, \theta, \phi) W(R \boldsymbol{E} \cdot \boldsymbol{N}) \sin \phi d R d \theta d \phi \\
= & \frac{\left(K^{(n)}-K^{(s)}\right) E_{i j} E_{s t}}{2} \int_{0}^{2 \pi} \int_{0}^{\pi / 2} \int_{R_{1}}^{R_{2}} D(R, \theta, \phi) R^{2} N_{i} N_{j} N_{s} N_{t} \sin \phi d R d \theta d \phi \\
& +\frac{K^{(s)} E_{i j} E_{s t}}{8} \int_{0}^{2 \pi} \int_{0}^{\pi / 2} \int_{R_{1}}^{R_{2}} D(R, \theta, \phi) R^{2}\left(\delta_{i s} N_{j} N_{t}+\delta_{i t} N_{j} N_{s}\right. \\
& \left.+\delta_{j s} N_{i} N_{t}+\delta_{j t} N_{i} N_{s}\right) \sin \phi d R d \theta d \phi .
\end{aligned}
$$

Here $D(R, \theta, \phi)$ is the distribution function of the positions in the upper hemispherical coordinate system which is located by the relative distance vectors, and reads

$$
D(R, \theta, \phi)=\sum_{I} \sum_{J} \sum_{K} \frac{M\left(R^{(I)}, \theta^{(J)}, \phi^{(K)}\right)}{2 V \sin \phi} \delta\left(R-R^{(I)}\right) \delta\left(\theta-\theta^{(J)}\right) \delta\left(\phi-\phi^{(K)}\right),
$$

where $I, J$ and $K$ extend over all the positions located by the distance vectors in the spherical coordinate system, respectively. $\delta$ is the Dirac delta function, and $M\left(R^{(I)}, \theta^{(J)}, \phi^{(K)}\right)$ is the number of the distance vectors locating at site $\left(R^{(I)}, \theta^{(J)}, \phi^{(K)}\right)$. In the spherical coordinates $N_{i}$ is represented as

$$
N_{1}=\sin \phi \cos \theta, \quad N_{2}=\sin \phi \sin \theta, \quad N_{3}=\cos \phi .
$$

Particularly, for isotropic assemblages of blocks with fully randomized relative distance vectors, the distribution function $D$ only depends on $R$. Making use of the following three integration formulae

$$
\begin{aligned}
& \alpha_{0}=\int_{R_{1}}^{R_{2}} D(R) R^{2} d R \\
& \int_{0}^{2 \pi} \int_{0}^{\pi / 2} N_{i} N_{j} \sin \phi d \theta d \phi=\frac{2 \pi}{3} \delta_{i j} \\
& \int_{0}^{2 \pi} \int_{0}^{\pi / 2} N_{i} N_{j} N_{s} N_{t} \sin \phi d \theta d \phi=\frac{2 \pi}{15}\left(\delta_{i j} \delta_{s t}+\delta_{i s} \delta_{j t}+\delta_{i t} \delta_{j s}\right)
\end{aligned}
$$

an analytical evaluation of Eq. (13) is given by

$$
e=\alpha_{0}\left(\frac{\pi}{15}\left(K^{(n)}-K^{(s)}\right)\left(\delta_{i j} \delta_{s t}+\delta_{i s} \delta_{j t}+\delta_{i t} \delta_{j s}\right)+\frac{\pi}{6} K^{(s)}\left(\delta_{i s} \delta_{j t}+\delta_{i t} \delta_{j s}\right)\right) E_{i j} E_{s t} .
$$

After substituting Eq. (17) into Eq. (12), we can obtain the tangent stiffness tensor

$$
C_{i j s t}=\frac{\pi}{15} \alpha_{0}\left(2\left(K^{(n)}-K^{(s)}\right)\left(\delta_{i j} \delta_{s t}+\delta_{i s} \delta_{j t}+\delta_{i t} \delta_{j s}\right)+5 K^{(s)}\left(\delta_{i s} \delta_{j t}+\delta_{i t} \delta_{j s}\right)\right) .
$$

Comparison of Eq. (18) and the standard isotropic linear elastic constitutive equation (LANDAU and LiFSHITZ, 1970) 


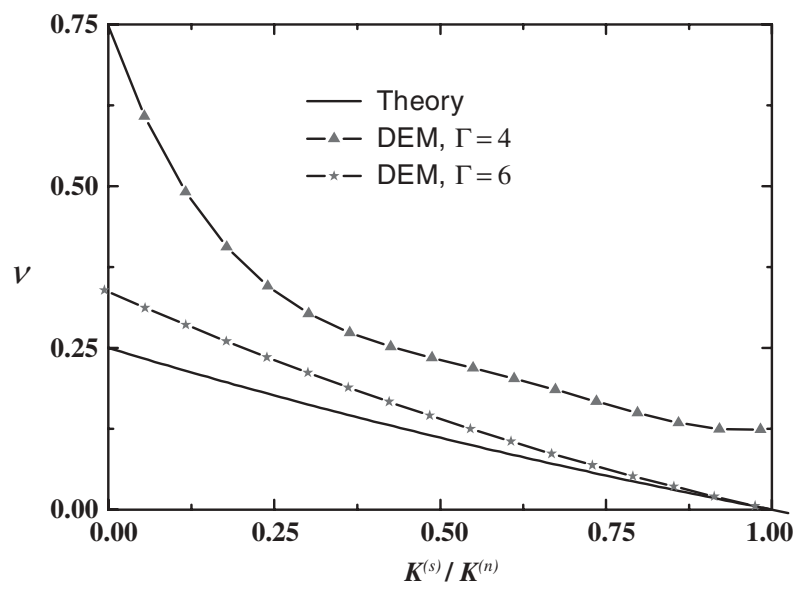

Figure 3

Showing the analytical results for the effect of contact stiffness ratio $K^{(s)} / K^{(n)}$ on effective Poisson ratio $v$ compared to the discrete element results.

$$
C_{i j s t}=\lambda \delta_{i j} \delta_{s t}+\mu\left(\delta_{i s} \delta_{j t}+\delta_{i t} \delta_{j s}\right)
$$

leads to the following equivalent relationships between the elastic constants $K^{(n)}, K^{(s)}$ and the macroscopic Yang's modulus $E$ and Poisson ratio $v$, respectively,

$$
K^{(n)}=\frac{3}{2 \pi \alpha_{0}} \frac{E}{1-2 v}, \quad K^{(s)}=\frac{3}{2 \pi \alpha_{0}} \frac{E(1-4 v)}{(1+v)(1-2 v)} .
$$

According to Eq. (20), the influence of the contact stiffness ratio $K^{(s)} / K^{(n)}$ on the macroscopic Poisson ratio $v$ is plotted in Figure 3. It shows that $v$ is zero when $K^{(s)}$ is equal to $K^{(n)}$. This is in agreement with the discrete element simulations for dense assemblies (the average number of contacts per particle $\Gamma=6$ ) (KRUYT and Rothenburg, 2004). The analytical data in Figure 3 also show that $v$ is not larger than 0.25 for positive shear stiffness. Although this is not very close to the numerical data, it should be noted that for most granular materials, Poisson ratio is in the range $0.2-0.25$. Therefore, we argue that for a statistical homogeneous rigid system, in which the interactions are concentrated at the contact points, the influence of the parameters $K^{(n)}, K^{(s)}$ on the macroscopic behaviour follows Eq. (20).

\subsection{Dissipation and Internal State Variables}

Irreversible nonlinear behavior of rock masses is the consequence of energy dissipation which is activated during the loading process, and can be traced largely to the contact breakage. In this paper, we assume that all damage dissipation in rocks results from a continuous process of the breakage of contact cohesive bonds between 
the blocks, and an internal variable characterizing the state of the bond between elements $a$ and $b$ at the present time $\tau$

$$
q^{(a, b)}= \begin{cases}0 & \text { The failure criterion has not been satisfied during loading process } \\ 1 & \text { The failure criterion has been satisfied during loading process }\end{cases}
$$

is introduced such that the breaking of this contact bond is modeled by irreversibly modifying the contact potential to zero when a certain breaking rule has been satisfied, i.e.,

$$
W^{(a, b)}=\left(1-q^{(a, b)}\right) W\left(R^{(a, b)} \boldsymbol{E} \cdot \boldsymbol{N}^{(a, b)}\right) .
$$

Consequently, the discrete summation form of the free energy is represented as

$$
e=\frac{1}{V} \sum_{<a, b>}\left(1-q^{(a, b)}\right) W\left(R^{(a, b)} \boldsymbol{E} \cdot \boldsymbol{N}^{(a, b)}\right)
$$

and its integral form is given by

$$
e=\int_{0}^{2 \pi} \int_{0}^{\pi / 2} \int_{R_{1}}^{R_{2}}\left(D(R, \theta, \phi)-D^{q}(R, \theta, \phi)\right) W(R \boldsymbol{E} \cdot \boldsymbol{N}) \sin \phi d R d \theta d \phi,
$$

where $D^{q}(R, \theta, \phi)$ is the distribution function of the positions in the upper hemispherical coordinate system which are located by all broken contact bonds at the current time.

Defining the energy functions on the orientation $(\theta, \phi)$ as

$$
\Phi(\theta, \phi, E \cdot N)=\int_{R_{1}}^{R_{2}} D(R, \theta, \phi) W(R \boldsymbol{E} \cdot \boldsymbol{N}) d R
$$

and extracting the damage variable on that orientation,

$$
d(\theta, \phi) \Phi(\theta, \phi, \boldsymbol{E} \cdot \boldsymbol{N})=\int_{R_{1}}^{R_{2}} D^{q}(R, \theta, \phi) W(R \boldsymbol{E} \cdot \boldsymbol{N}) d R
$$

We introduce the general form of the Helmholtz free energy which is sufficient to describe all reversible processes in rock masses. The total free energy per unit volume depends on the observable variable $\boldsymbol{E}$ as well as the damage variable $d(\theta, \phi)$, and takes the following compact form

$$
e=\int_{\Omega}\left(1-d^{\Omega}\right) \Phi^{\Omega}(\boldsymbol{E} \cdot \boldsymbol{N}) d \Omega
$$

Applying the Clausius-Duhem inequality to the derived free energy

$$
\sigma_{i j} \dot{E}_{i j}-\dot{e} \geq 0
$$


we arrive according to Eq. (27) at

$$
\dot{E}_{i j}\left(\sigma_{i j}-\int_{\Omega}\left(1-d^{\Omega}\right) \frac{\partial \Phi^{\Omega}(\boldsymbol{E} \cdot \boldsymbol{N})}{\partial E_{i j}} d \Omega\right)+\int_{\Omega} \dot{d}^{\Omega} \Phi^{\Omega}(\boldsymbol{E} \cdot \boldsymbol{N}) d \Omega \geq 0 .
$$

This inequality must hold for arbitrary strain-rates. Therefore we can conclude that Eq. (29) and the following statements are equivalent:

- The stress strain relationship

$$
\sigma_{i j}=\int_{\Omega}\left(1-d^{\Omega}\right) \frac{\partial \Phi^{\Omega}(\boldsymbol{E} \cdot \boldsymbol{N})}{\partial E_{i j}} d \Omega
$$

from which the expression for the strain-rates results again by time differentiation

$$
\dot{\sigma}_{i j}=\dot{E}_{s t} \int_{\Omega}\left(1-d^{\Omega}\right) \frac{\partial^{2} \Phi^{\Omega}(\boldsymbol{E} \cdot \boldsymbol{N})}{\partial E_{i j} \partial E_{s t}} d \Omega-\int_{\Omega} \dot{d}^{\Omega} \frac{\partial \Phi^{\Omega}(\boldsymbol{E} \cdot \boldsymbol{N})}{\partial E_{i j}} d \Omega .
$$

- The damage dissipation inequality

$$
\int_{\Omega} \dot{d}^{\Omega} \Phi^{\Omega}(\boldsymbol{E} \cdot \boldsymbol{N}) d \Omega \geq 0
$$

Based on the fact that the energy stored in the contact cohesive bonds is positive and also, that the dissipative energy gradually increases during the damage processes, the definitions as shown in Eq. (26) apparently ensure that the damage dissipation inequality always holds on each orientation. In other words, the Clausius-Duhem inequality (28) is naturally fulfilled in the course of the physical choice of the damage variables.

Because accurate description of the local effects of the defects are not available for the material under investigation, at the present time information on $d^{\Omega}$ and its evolution has to depend on the development of recovery experiments. On the other hand, the orientation-independent nature of the free energy function $\Phi^{\Omega}$ in Eq. (32) motivates us to assume that the damage dissipation also evolves independently on each orientation, thus identifying $\Phi^{\Omega}$ as the thermodynamic force conjugate to $d^{\Omega}$. It should be noted that this basic assumption overlaps with certain concepts in which multiple-plane representations of inelasticity are derived (SEAmAn and Dein, 1983; BAŽAnt and Gambarova, 1984; Ju and LeE, 1991a,b; EsPinosa and Brar, 1995; PEnsÉE et al., 2002).

In the context of the irreversible thermodynamics theory (LEMAITRE, 1992; MAUGin, 1992), a general form of the damage evolution equations on a generic orientation can consequently be formulated through a dissipation potential function in the space of $\Phi^{\Omega}$ in combination with the maximum dissipation principle (SIMO and Hughes, 1998). The detailed expressions are given as follows: 
- Define a damage potential function $\pi^{\Omega}\left(\Phi^{\Omega}, d^{\Omega}\right)$ on a generic orientation such that this damage potential function coincides with the damage surface $\pi^{\Omega}\left(\Phi^{\Omega}, d^{\Omega}\right)=0$ on that orientation. To ensure that the damage evolution follows the path in the damage variable space that maximizes the dissipation on that orientation, we gain the evolution law

$$
\dot{d}^{\Omega}=\dot{\lambda}^{\Omega} \frac{\partial \pi^{\Omega}\left(\Phi^{\Omega}, d^{\Omega}\right)}{\partial \Phi^{\Omega}},
$$

where parameter $\dot{\lambda}_{\Omega}$ is determined from the consistency condition $\dot{\pi}_{\Omega}=0$ :

$$
\dot{\lambda}^{\Omega}=-\dot{\Phi}^{\Omega} / \frac{\partial \pi^{\Omega}\left(\Phi^{\Omega}, d^{\Omega}\right)}{\partial d^{\Omega}} .
$$

- The loading/unloading conditions are defined by the condition that each of the following Kuhn-Tucker relations always should be satisfied (JU, 1990)

$$
\dot{\lambda}^{\Omega} \geq 0, \quad \pi^{\Omega}\left(\Phi^{\Omega}, d^{\Omega}\right) \leq 0, \quad \dot{\lambda}^{\Omega} \pi^{\Omega}\left(\Phi^{\Omega}, d^{\Omega}\right)=0 .
$$

Given the evolution equations of $d^{\Omega}$ on a generic orientation, the overall responses of brittle materials result from the cumulative effect of processes taking place on all possible orientations in the upper hemisphere. Therefore, Eqs. (27), (30), (31), (33), (34) and (35) furnish a closed constitutive formulation for rocks, which we identify as the orientation-based continuum damage model in this paper. As a consequence of the independent evolution of dissipation processes on different orientations, the mechanical behavior of materials generally becomes anisotropic characteristics in the inelastic regime even if the material is initially isotropic, thus introducing damageinduced anisotropy in a very natural and simple way.

\section{Lattice Solid Model}

Subdivide the upper hemispherical envelop into $M$ discrete contiguous solid angles, $\Delta \boldsymbol{\Omega}^{(m)}$, each associated with a corresponding discrete orientation $N_{i}^{(m)}$. The total energy of the infinitesimal neighborhood of the material point can thus be estimated approximately by the sum of a finite number of discrete components defined on $M$ orientations, i.e.,

$$
V e=V \int_{\Omega}\left(1-d^{\Omega}\right) \Phi^{\Omega}(\boldsymbol{E} \cdot \boldsymbol{N}) d \Omega \approx \sum_{m=1}^{M}\left(1-d^{(m)}\right) \Phi^{(m)}\left(\boldsymbol{E} \cdot \boldsymbol{N}^{(m)}\right) V \Delta \boldsymbol{\Omega}^{(m)} .
$$

Because both the evolution of damage variables $d^{(m)}$ and the calculation for the free energy functions $\Phi^{(m)}$ are independent of the orientations, we can analogously construct a lattice cell with the total of $M$ rod elements, in which the $m$-th rod element with orientation $N_{i}^{(m)}$, cross section $A^{(m)}$ and length $l^{(m)}$ makes the 
deformation compatible with $\boldsymbol{E} \cdot \boldsymbol{N}^{(m)}$, obeys the evolution laws of $d^{(m)}$, and has the energy

$$
\left(1-d^{(m)}\right) \Phi^{(m)}\left(\boldsymbol{E} \cdot \boldsymbol{N}^{(m)}\right) A^{(m)} l^{(m)} .
$$

In this way, once the following equation

$$
V \Delta \Omega^{(m)}=A^{(m)} l^{(m)}
$$

holds, the infinitesimal neighborhood of the material point can be replaced with the energy equivalent lattice cell. This counterpart is considerably coarser than the characteristic length of the block, but can approximately perform the macroscopic response of the assemblage of blocks with the enormously large number of degrees of freedom under the macroscopic strain $\boldsymbol{E}$.

For example, we estimate the energy using 13 integration points oriented in $\{1,0,0\}+\{1,1,0\}+\{1,1,1\}$ directions on the envelope of the upper hemisphere, and the values of the corresponding solid angles are given by

$$
\begin{aligned}
& \Delta \Omega^{[1,0,0]}=\Delta \Omega^{[0,1,0]}=\Delta \Omega^{[0,0,1]}=\frac{10}{51} \pi \\
& \Delta \Omega^{[1,1,0]}=\Delta \Omega^{[1,0,1]}=\Delta \Omega^{[0,1,1]}=\Delta \Omega^{[1, \overline{1}, 0]}=\Delta \Omega^{[\overline{1}, 0,1]}=\Delta \Omega^{[0, \overline{1}, 1]}=\frac{12}{85} \pi \\
& \Delta \Omega^{[1,1,1]}=\Delta \Omega^{[\overline{1}, 1,1]}=\Delta \Omega^{[1, \overline{1}, 1]}=\Delta \Omega^{[\overline{1}, \overline{1}, 1]}=\frac{12}{85} \pi
\end{aligned}
$$

It can be proved that this 13-point formula can satisfy the zeroth moment constraint, i.e.,

$$
\begin{aligned}
& \int_{\Omega} d \boldsymbol{\Omega}=\sum_{m=1}^{13} \Delta \boldsymbol{\Omega}^{(m)} \\
& \int_{\Omega} N_{i} N_{j} d \boldsymbol{\Omega}=\sum_{m=1}^{13} N_{i}^{(m)} N_{j}^{(m)} \Delta \boldsymbol{\Omega}^{(m)} \\
& \int_{\Omega} N_{i} N_{j} N_{S} N_{t} d \Omega=\sum_{m=1}^{13} N_{i}^{(m)} N_{j}^{(m)} N_{s}^{(m)} N_{t}^{(m)} \Delta \boldsymbol{\Omega}^{(m)}
\end{aligned}
$$

Correspondingly, a cubic lattice cell is constructed, which consists of 13 rod elements oriented in $\{1,0,0\}+\{1,1,0\}+\{1,1,1\}$ directions, as shown in Figure 4(a). Let the side length of the cubic be $a$. The lengths of the rod elements are $a, a \sqrt{2}$ and $a \sqrt{3}$, respectively.

In particular, for isotropic assemblages of blocks, the energy of the $m$-th rod element is derived from Eqs. (13), (16), (20), (37) and (38) as

$$
\frac{a^{3} \Delta \Omega^{(m)}}{2\left(l^{(m)}\right)^{2}}\left[\frac{3}{2 \pi} \frac{E}{1-2 v} \delta u_{i}^{(m)} \delta u_{i}^{(m)}+\frac{3}{2 \pi} \frac{E(1-4 v)}{(1+v)(1-2 v)} \delta v_{i}^{(m)} \delta v_{i}^{(m)}\right],
$$


(a)
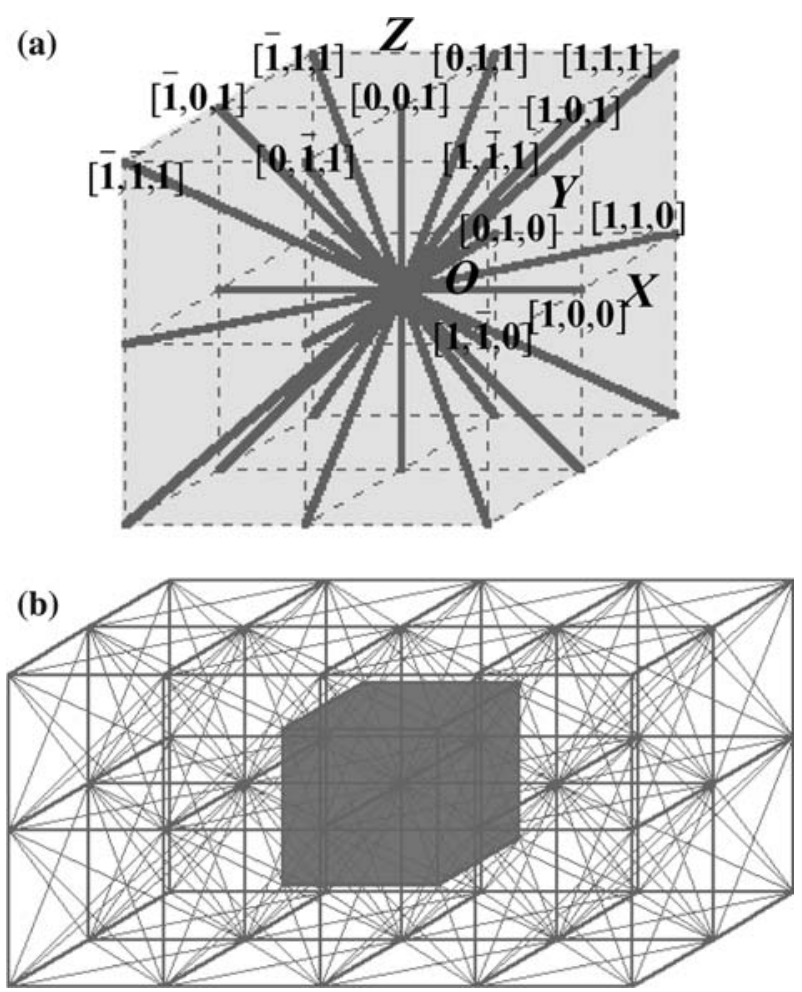

Figure 4

(a) Three-dimensional regular lattice unit cell; (b) the periodic lattices made up of the unit cell.

where

$$
\delta u_{i}^{(m)}=l^{(m)} E_{s t} N_{i}^{(m)} N_{s}^{(m)} N_{t}^{(m)}, \quad \delta v_{i}^{(m)}=l^{(m)} E_{i j} N_{j}^{(m)}-\delta u_{i}^{(m)}
$$

As a result, the elastic normal stiffness $K_{n}^{(m)}$ and the shear stiffness $K_{s}^{(m)}$ of the rod element with orientation $N_{i}^{(m)}$ have the following format

$$
k_{n}^{(m)}=\frac{3 E a^{3} \Delta \Omega^{(m)}}{2 \pi\left(l^{(m)}\right)^{2}(1-2 v)}, \quad k_{s}^{(m)}=\frac{3 E a^{3} \Delta \Omega^{(m)}(1-4 v)}{2 \pi\left(l^{(m)}\right)^{2}(1+v)(1-2 v)} .
$$

Taking this three-dimensional lattice cell as the unit cell of a periodic discrete lattice shown in Figure 4(b), the constructed lattice solid model can be conceived as an alternative manner of the numerical implementation of the orientation-based constitutive model, instead of the finite-element method. 


\section{Conclusions}

We present a multiscale modeling methodology to describe the transition from micro to macro domain of rock, and formulate the Helmholtz free energy for a non-dissipative case.

By physically introducing the damage variables into the Helmholtz free energy, we construct the orientation-based continuum damage model, which is highly promising for the solution of engineering problems involving a damage process with a greatly simplified mathematical description.

The discretization of the orientation-based constitutive model furnishes a unified framework for the development of the lattice solid model.

\section{Acknowledgements}

We gratefully acknowledge the support from National Natural Science Foundation of China under grant Nos. 10232050 and 10572140, Special Funds for Major state Basic Research Project under Grant Nos. 2002CB412703 and 2002CB412706.

\section{REFERENCES}

BATHURST, R.J. and RothenBuRG, L. (1988), Micromechanical aspects of isotropic granular assemblies with linear contact interactions, J. Appl. Mech. 55, 17-23.

BAŽAnt, Z. and Gambarova. P. (1984), Crack shear in concrete: Crack band microplane model, ASCE J. Eng. Mech. 110, 2015-2035.

Christofferson, J., Mehrabadi, M.M., and Nemat-Nasser, S. (1981), A micromechanical description of granular material behavior, J. Appl. Mech. 48, 339-344.

Espinosa, H.D. and BRAR, N.S. (1995), Dynamic failure mechanisms of ceramic bars: experiments and numerical simulations, J. Mech. Phys. Solids. 43, 1615-1638.

Fung, Y.C., Foundations of Solid Mechanics (Prentice, New Jersey 1969).

JING, L. (1998), Formulations of discontinuous deformation analysis for block systems, Eng. Geology. 49, $371-381$.

JU, J.W. (1990), Isotropic and anisotropic damage variable in continuum damage mechanics, ASCE J. Eng. Mech. 116, 2764-2770.

Ju, J.W. and LEE, X. (1991a), On three-dimensional self-consistent micromechanical damage models for brittle solids, Part I: Tensile loadings, ASCE J. Eng. Mech. 117, 1495-1514.

JU, J.W. and LEE, X. (1991b), On three-dimensional self-consistent micromechanical damage models for brittle solids, Part II: Compressive loadings, ASCE J. Eng. Mech. 117, 1515-1536.

Kruyt, N.P. and Rothenburg, L. (2004), Kinematic and static assumptions for homogenization in micromechanics of granular materials, Mech. Mater. 36, 1157-1173.

Landau, L.D. and Lifshitz, E.M., Theory of Elasticity (Pergamon, Oxford 1970).

Lemaitre, J., A Course on Damage Mechanics (Springer, Berlin 1992).

LI, L.H., BAI, Y.L., XIA, M.F., KE, F.J., and YIN, X.C. (2000), Damage localization as a possible precursor of earthquake rupture, Pure Appl. Geophys. 157, 1929-1943.

Maugin, G.A., The Thermomechanics of Plasticity and Fracture (Cambridge University Press, Cambridge 1992).

Mora, P. and Place D. (1999), The weakness of earthquake faults, Geophys. Res. Lett. 26, $123-126$. 
NAPIER, J.A.L. and DEDE, T.A. (1997), Comparison between random mesh schemes and explicit growth rules for rock fracture simulation, Int. J. Rock Mech. Min. Sci. 34, 356.

Pensée, V., Kondo, D., and Dormieux, L. (2002), A micromechanical analysis of anisotropic damage in brittle materials, ASCE J. Eng. Mech. 128, 889-897.

Seaman, L. and Dein, J.L., Representing shear band damage at high strain rates. In Proc. IUTAM Symposium on Nonlinear Deformation Waves (ed. Tallin, Estonia) (Springer-Verlag, Berlin 1983).

Simo, J.C. and Hughes, T.J.R., Computational inelasticity. In Interdisciplinary Applied Mathematics (Springer, New York 1998).

Walton, K. (1987), The effective elastic moduli of a random packing of spheres, J. Mech. Phys. Solids. 35 , $213-226$.

(Received November 17, 2004, revised December 23, 2005, accepted December 28, 2005)

To access this journal online:
http://www.birkhauser.ch

